\title{
Laser-spectroscopy studies of the nuclear structure of neutron-rich radium
}

\author{
K. M. Lynch, ${ }^{1, *}$ S. G. Wilkins, ${ }^{2}$ J. Billowes, ${ }^{2}$ C. L. Binnersley, ${ }^{2}$ M. L. Bissell, ${ }^{2}$ K. Chrysalidis,${ }^{3,4}$ T. E. Cocolios,${ }^{5}$ \\ T. Day Goodacre, ${ }^{2,3, \dagger}$ R. P. de Groote, ${ }^{5}$ G. J. Farooq-Smith, ${ }^{5}$ D. V. Fedorov, ${ }^{6}$ V. N. Fedosseev,${ }^{3}$ K. T. Flanagan, ${ }^{2}$ S. Franchoo, ${ }^{7}$ \\ R. F. Garcia Ruiz, ${ }^{2}$ W. Gins, ${ }^{5}$ R. Heinke, ${ }^{4}$ Á. Koszorús, ${ }^{5}$ B. A. Marsh, ${ }^{3}$ P. L. Molkanov,${ }^{6}$ P. Naubereit ${ }^{4}$ G. Neyens, ${ }^{5}$ \\ C. M. Ricketts, ${ }^{2}$ S. Rothe, ${ }^{3}$ C. Seiffert, ${ }^{3}$ M. D. Seliverstov, ${ }^{4}$ H. H. Stroke, ${ }^{8}$ D. Studer, ${ }^{4}$ A. R. Vernon, ${ }^{2}$ \\ K. D. A. Wendt, ${ }^{4}$ and X. F. Yang ${ }^{5}$ \\ ${ }^{1}$ ISOLDE, EP Department, CERN, CH-1211 Geneva-23, Switzerland \\ ${ }^{2}$ School of Physics and Astronomy, The University of Manchester, Manchester, M13 9PL, United Kingdom \\ ${ }^{3}$ EN Department, CERN, CH-1211 Geneva 23, Switzerland \\ ${ }^{4}$ Institut für Physik, Johannes Gutenberg-Universität Mainz, D-55128 Mainz, Germany \\ ${ }^{5}$ KU Leuven, Instituut voor Kern- en Stralingsfysica, B-3001 Leuven, Belgium \\ ${ }^{6}$ Petersburg Nuclear Physics Institute, 188300 Gatchina, Russia \\ ${ }^{7}$ Institut de Physique Nucléaire d'Orsay, F-91406 Orsay, France \\ ${ }^{8}$ Department of Physics, New York University, New York, New York 10003, USA
}

(Received 27 September 2017; published 7 February 2018)

\begin{abstract}
The neutron-rich radium isotopes, ${ }^{222-233} \mathrm{Ra}$, were measured with Collinear Resonance Ionization Spectroscopy (CRIS) at the ISOLDE facility, CERN. The hyperfine structure of the $7 s^{2}{ }^{1} S_{0} \rightarrow 7 s 7 p^{3} P_{1}$ transition was probed, allowing measurement of the magnetic moments, quadrupole moments, and changes in mean-square charge radii. These results are compared to existing literature values, and the new moments and change in mean-square charge radii of ${ }^{231} \mathrm{Ra}$ are presented. Low-resolution laser spectroscopy of the very neutron-rich ${ }^{233} \mathrm{Ra}$ has allowed the isotope shift and relative charge radius to be determined for the first time.
\end{abstract}

DOI: 10.1103/PhysRevC.97.024309

\section{INTRODUCTION}

The neutron-rich radium isotopes are located in a region of dynamic nuclear structure. Above the $N=126$ shell closure in the $220 \leqslant A \leqslant 228$ mass range, a region of octupole deformation is present [1,2], famously evidenced with the "pear-shaped" form of ${ }^{224} \mathrm{Ra}$ [3]. Past the border of reflection asymmetry around $N=140, \beta_{3}$ is predicted to return to zero [4], and purely quadrupole deformation is expected to resume. However, despite the reflection symmetric shapes, octupole correlations have been discovered to persist in ${ }^{227,229} \mathrm{Ra}$ [5,6] and, to a lesser extent, ${ }^{231} \mathrm{Ra}$ [7]. Systematic studies across the neutron-rich radium isotopic chain are necessary to characterize the nature of the (quadrupole and octupole) deformation and study the effects of these persistent octupole correlations.

Laser spectroscopy provides a nuclear-model independent measurement of nuclear observables; most notably for these studies, the quadrupole moment and change in mean-square

\footnotetext{
*kara.marie.lynch@cern.ch

${ }^{\dagger}$ Present address: TRIUMF, Vancouver, British Columbia, V6T 2A3, Canada.

Published by the American Physical Society under the terms of the Creative Commons Attribution 4.0 International license. Further distribution of this work must maintain attribution to the author(s) and the published article's title, journal citation, and DOI.
}

charge radii between isotopes. Initial laser-spectroscopy experiments studying radium measured the hyperfine structure of atomic $(\mathrm{Ra})$ and ionic $\left(\mathrm{Ra}^{+}\right)$radium isotopes [8,9] for five different transitions. In the case of the alkali-metallike $\mathrm{Ra}^{+}$ions, the single valence electron simplified the $a b$ initio calculations of the field- and mass-shift factors for extraction of the change in mean-square charge radii from the measured isotope shifts. More recent measurements of the isotope shifts of $\mathrm{Ra}^{+}$have arisen from a desire for a detailed knowledge of the atomic structure of radium to aid atomic parity nonconservation (APNC) tests $[10,11]$. An atomic test of the standard model, APNC gives rise to a small parity nonconserving electric dipole transition amplitude $\left(E 1_{\mathrm{PNC}}\right)$ that can be measured in the $7 s^{2} S_{1 / 2} \rightarrow 6 d^{2} D_{3 / 2}$ transition with a singly trapped radium ion $[12,13]$. This paper takes advantage of the recent $a b$ initio many-body calculations, motivated by the APNC work, that provide new values for the field shifts and specific mass shifts of the neighboring levels in the radium atoms and ions [14].

Expanding upon the laser-spectroscopy measurements already performed, the hyperfine structure of the atomic transition $7 s^{21} S_{0} \rightarrow 7 s 7 p^{3} P_{1}(714 \mathrm{~nm})$ was studied, allowing measurements of the nuclear spins, electromagnetic moments, and isotope shifts of the neutron-rich radium isotopes. Measurements were performed for the isotopes $222 \leqslant A \leqslant 232$ in high resolution, providing new values for the isotope shifts of ${ }^{227-232} \mathrm{Ra}$ for the 714-nm transition, in addition to the electromagnetic moments of the neutron-rich ${ }^{231} \mathrm{Ra}$. Low-resolution laser-spectroscopy measurements were also performed for 
${ }^{233} \mathrm{Ra}$, allowing the isotope shift to be determined. Very good agreement is found between our values and previous values $[8,9,14-16]$ for all nuclear observables measured.

The chain of 12 studied neutron-rich isotopes provide the ability to investigate the intrinsic quadrupole moment as neutrons fill the $v\left(2 g_{9 / 2}\right)$ and $v\left(1 i_{11 / 2}\right)$ orbitals past the $N=126$ shell closure. A surprisingly linear trend for the intrinsic quadrupole moments is exhibited, irrespective of filled orbitals or the presence of octupole deformation. Additionally, the odd-even staggering of the isotope shift (or change in mean-square charge radii) between neighboring isotopes offers further insight into the border of reflection asymmetry and the effect of the diminishing octupole correlations as ${ }^{231} \mathrm{Ra}$ is reached.

\section{EXPERIMENTAL TECHNIQUE}

The radium isotopes were produced at the ISOLDE facility by impinging 1.4-GeV protons onto a thick uranium-carbide target. After effusing and diffusing out of the target material, the radium atoms were ionized inside a high temperature $\left(\approx 2000^{\circ} \mathrm{C}\right)$ tantalum cavity by a combination of surface ionization and multistep laser resonance ionization [using the resonance ionization laser ion source (RILIS)]. They were then accelerated to $30 \mathrm{keV}$ and mass separated with the highresolution separator. The ions were then cooled and bunched using the ISOLDE linear Paul trap, ISCOOL, re-accelerated to $30 \mathrm{keV}$ and deflected through the CRIS beamline $[19,20]$. After passing the $\mathrm{Ra}^{+}$beam through a potassium-vapor chargeexchange cell for neutralization (with an efficiency of up to $60 \%$ ), the remaining ionic component was deflected away. The atom bunch was then spatially and temporally overlapped with pulsed light from two lasers (see Fig. 1) for resonance excitation and ionization. This was performed in an $1.2-\mathrm{m}$ "interaction region" held at a pressure of $1.4 \times 10^{-9}$ mbar.

The atoms were resonantly excited with 714.319-nm laser light $\left(13999.3569 \mathrm{~cm}^{-1}\right)$ using the $7 s^{21} S_{0} \rightarrow 7 s 7 p^{3} P_{1}$ atomic transition. Continuous-wave (cw) laser light at $714 \mathrm{~nm}$ was produced by a Matisse 2 TS titanium:sapphire (Ti:Sa) ring laser, pumped by 532-nm cw light produced by a Verdi Nd:YVO4 laser. The cw light was then "chopped" into 200-ns pulses at a repetition rate of $100 \mathrm{~Hz}$ by passing the light through a fast-switching Pockels cell [21]. This chopping of the cw laser beam was achieved by inducing a polarization change of $\lambda / 2$ in the Pockels cell with a Behlke FSWP91-01 fast square-wave pulser ( $0 \mathrm{kV}$ off, $2.4 \mathrm{kV}$ on). This approach was necessary to avoid optical pumping to dark sub-states (see Sec. III A for further discussion). It also enabled the temporal separation of the laser light for the 714-nm spectroscopic transition with the light for the subsequent excitation and ionization. The 714-nm laser light was then mirror coupled and deflected along the central axis of the CRIS beamline. The following steps used two-step ionization into the continuum from the $7 s 7 p^{3} P_{1}$ state with 555-nm laser light, with a pulse width of $10 \mathrm{~ns}$, delayed by $200 \mathrm{~ns}$ after the start of the 714-nm laser light pulse. This delay provided the optimum line shape and resolution for the hyperfine structure spectra [22]. The second step was a resonant excitation from the $7 s 7 p^{3} P_{1}$ to the $7 s 7 d^{3} D_{2}$ state at $31993.40 \mathrm{~cm}^{-1}$, followed by nonresonant ionization to

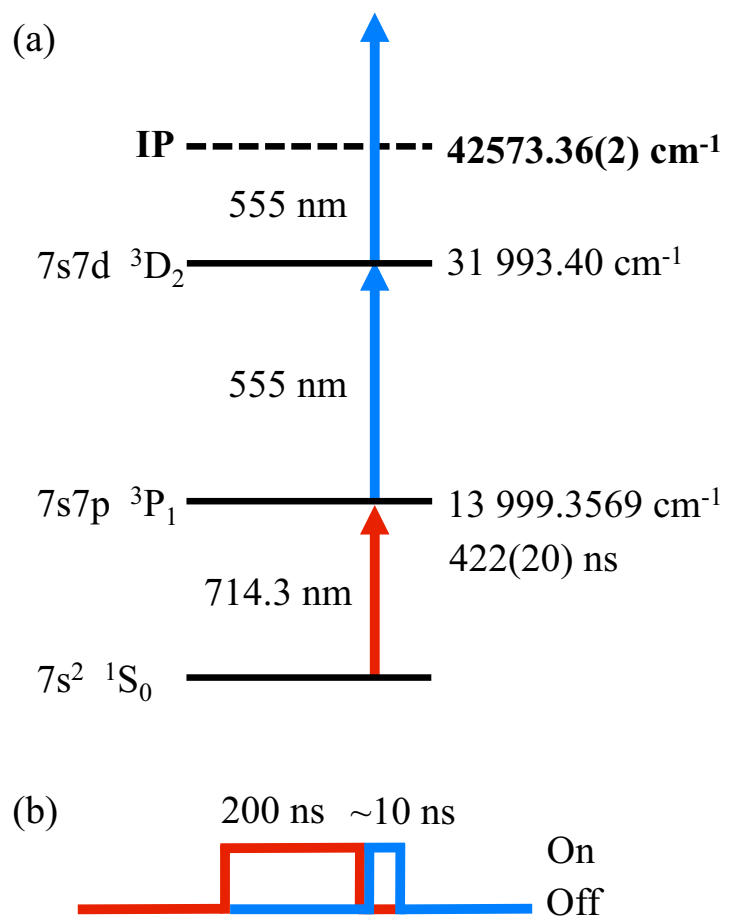

FIG. 1. (a) The resonance ionization scheme used to measure the hyperfine structure of the neutron-rich radium isotopes [17,18]. (b) The time structure of the 714-nm and 555-nm laser light pulses.

the continuum. The 555-nm laser light was produced by a Spectron Spectrolase 4000 pulsed-dye laser pumped by a Litron LPY 601 50-100 PIV Nd:YAG laser at a repetition rate of $100 \mathrm{~Hz}$. A schematic of the resonance ionization scheme is shown in Fig. 1(a), with the time structure of the "chopped" 714-nm and pulsed 555-nm laser light shown in Fig. 1(b). The hyperfine structure of the 714-nm transition was probed with a linewidth of down to $30 \mathrm{MHz}$. Broadband 714-nm light was also produced by pumping a Ti:Sa Z cavity [23] with 532-nm light from a Photonics Industries DM60 Nd:YAG laser. This produced laser light with a $>5-\mathrm{GHz}$ linewidth, which was used to search for the hyperfine-structure resonances and measure the low-yield isotope ${ }^{233} \mathrm{Ra}$. This isotope could not be measured with high resolution because of time considerations. The timing of the laser-light pulses was synchronized with the release of the ion bunch from ISCOOL by a Quantum Composers 9528 digital delay pulse generator, at a rate of $100 \mathrm{~Hz}$. The laser-ionized ions were deflected through $20^{\circ}$ and detected by a Hamamatsu F-4655-12 microchannel plate, biased at $-2.4 \mathrm{kV}$. The combined transmission and detection efficiency of the microchannel plate was determined to be $32 \%$ [20].

\section{RESULTS AND DISCUSSION}

\section{A. The hyperfine structure}

The high-resolution hyperfine-structure spectra of the $7 s^{21} S_{0} \rightarrow 7 s 7 p^{3} P_{1}$ transition in the neutron-rich radium isotopes are shown in Fig. 2. The frequency is shown relative to the centroid frequency of the reference isotope ${ }^{226} \mathrm{Ra}$. 


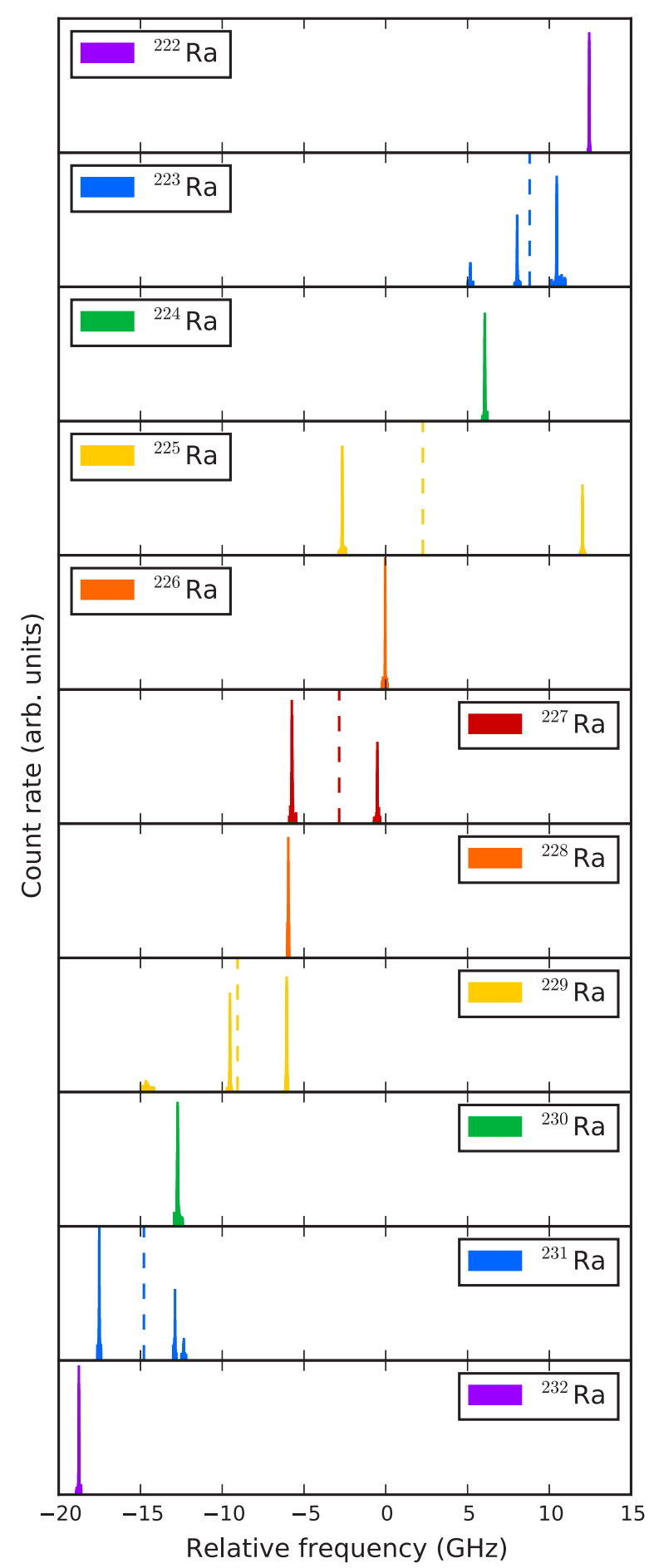

FIG. 2. Hyperfine structures of the neutron-rich radium isotopes ${ }^{222-232} \mathrm{Ra}$ measured by collinear resonance ionization spectroscopy, relative to the centroid frequency of the reference isotope ${ }^{226} \mathrm{Ra}$. The dotted lines illustrate the weighted centroids for the odd- $A$ isotopes. The $y$ axis displays arbitrary units that are different for each isotope.

The most neutron-rich isotope ${ }^{233} \mathrm{Ra}$ was only measured in low resolution. In Fig. 3 (top) the newly measured (highresolution) hyperfine structure of ${ }^{231} \mathrm{Ra}$ is compared to the (low-resolution) measurement of ${ }^{233} \mathrm{Ra}$ (bottom), from which
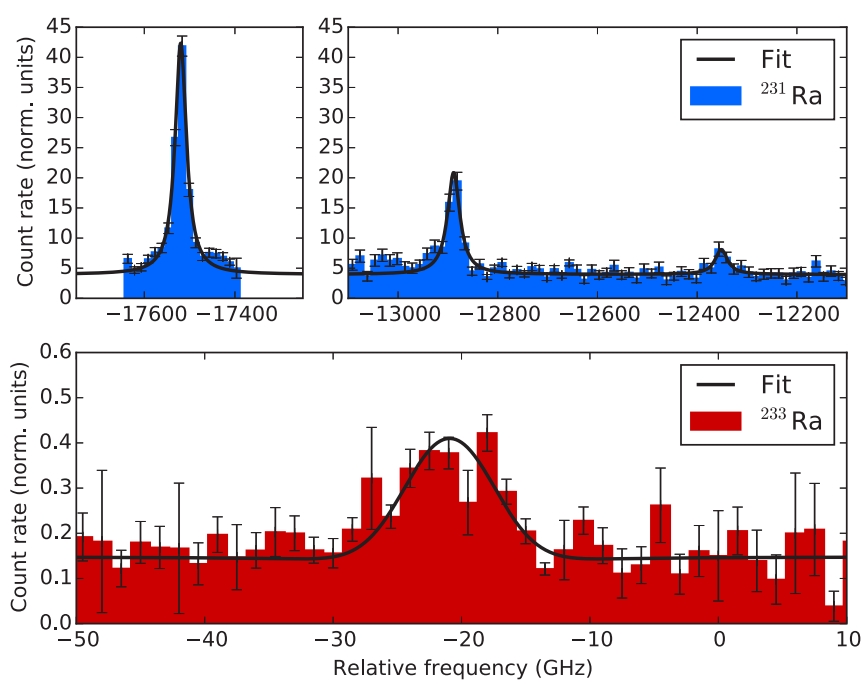

FIG. 3. Hyperfine structure of (top) ${ }^{231} \mathrm{Ra}$ in high resolution, and (bottom) ${ }^{233} \mathrm{Ra}$ in low resolution, both plotted relative to the centroid frequency of the reference isotope ${ }^{226} \mathrm{Ra}$.

the change in its relative charge radius only can be extracted. Each hyperfine structure was fit to a pseudo-Voigt line shape using a $\chi^{2}$-minimization routine to extract $A\left({ }^{3} P_{1}\right), B\left({ }^{3} P_{1}\right)$ and the centroid frequency $v^{A}$.

The even-even isotopes in Fig. 2 show the typical singlepeak structure resulting from their $0^{+}$nuclear spin. The odd- $A$ isotopes in Fig. 2 have either two (for spin $I=1 / 2$ isotopes) or three (for $I>1 / 2$ isotopes) hyperfine-structure peaks. The third peak in the hyperfine structure of ${ }^{227} \mathrm{Ra}$ was not measured, and the intensity of the $F_{I} \rightarrow F_{I-1}^{\prime}$ peaks in the hyperfine structures of ${ }^{229,231} \mathrm{Ra}$ are smaller than expected from angular-momentum coupling considerations. This is suggested to be from (despite chopping the laser light) the linearly polarized $\mathrm{cw}$ light optically pumping the atoms into the $m_{F}=m_{ \pm I}$ substates of the ${ }^{1} S_{0}$ atomic state. These "dark" substates reduce the population available to be excited to the $F_{I-1}^{\prime}$ upper state, thus reducing the intensity of the hyperfine structure peak. To fit the hyperfine spectra of ${ }^{227} \mathrm{Ra}$, the ratio $B\left({ }^{1} P_{1}\right) / B\left({ }^{3} P_{1}\right)=-0.896(5)$ was used, experimentally determined for the isotopes ${ }^{221,223} \mathrm{Ra}[8]$.

The nuclear spins $I$ and isotope shifts $\left(\delta v^{\text {ref, A }}=v^{A}-v^{\text {ref }}\right)$ of the neutron-rich radium isotopes are presented in Table I. For completeness, column 3 presents the isotope shifts relative to the reference isotope ${ }^{226} \mathrm{Ra}$ and column 4 presents the isotope shifts relative to ${ }^{214} \mathrm{Ra}$ at the $N=126$ shell closure, using $\delta v^{226,214}=+41744(4) \mathrm{MHz}$ measured from this work. The centroid of the closest-in-time reference scan was used to calculate the isotope shift for each isotope. For ${ }^{231} \mathrm{Ra}$, the isotope with the largest data set, a standard deviation of $3 \mathrm{MHz}$ on the isotope shifts was present. Therefore, a systematic uncertainty of $3 \mathrm{MHz}$ was assigned to each $\delta v^{226, A}$ isotope shift, to account for the scatter over the course of the experiment. For isotopes with multiple scans, the weighted mean and weighted standard deviation of $A\left({ }^{3} P_{1}\right)$ and $B\left({ }^{3} P_{1}\right)$ were calculated. Literature values of the 714-nm transition isotope shifts are taken from Ref. [8]. 
TABLE I. Isotope shifts for the $7 s^{21} S_{0} \rightarrow 7 s 7 p^{3} P_{1}$ transition and change in mean-square charge radii for the neutron-rich radium isotopes, ${ }^{222-233} \mathrm{Ra}$. Statistical and systematic uncertainties on the mean-square charge radii are presented in parentheses and brackets, respectively. $\Delta$ values are used to scale the change in mean-square charge radii to account for nuclear deformation [14]. $\Delta$ values for ${ }^{231,233}$ Ra are estimated.

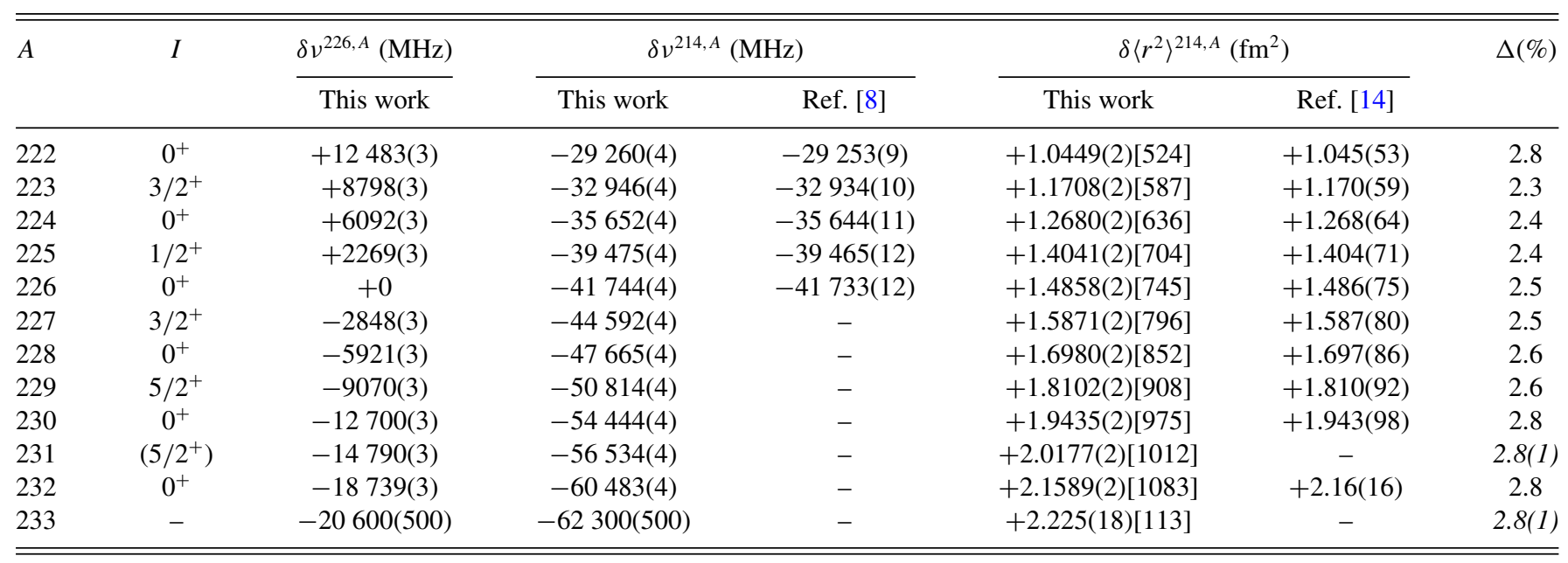

The isotope shift for the low-resolution scans of ${ }^{233} \mathrm{Ra}$, presented in Fig. 3 (bottom), was determined in a similar way. The standard deviation (scatter) was calculated to be $500 \mathrm{MHz}$ and assigned to the isotope shift of ${ }^{233} \mathrm{Ra}$. Because of its very neutron-rich nature, many properties of ${ }^{233} \mathrm{Ra}$, including the nuclear spin, are not known. As the linewidth of the laser was too large to resolve any underlying structure, the hyperfine structure peak was fitted as a single peak (the $A$ and $B$ factors set to zero) and only the isotope shift was extracted.

\section{B. King-plot analysis}

The changes in mean-square charge radii $\delta\left\langle r^{2}\right\rangle^{A, A^{\prime}}$ between isotopes were evaluated from the measured isotope shifts using

$$
\delta v_{714}^{A, A^{\prime}}=F_{714} \delta\left\langle r^{2}\right\rangle^{A, A^{\prime}}+M_{714} \frac{A^{\prime}-A}{A A^{\prime}},
$$

where $F$ and $M$ are the field- and mass-shift factors, respectively. ${ }^{1}$ For consistency with our previous analyses, and to allow our results to be directly comparable, $\lambda^{A, A^{\prime}} \approx \delta\left\langle r^{2}\right\rangle^{A, A^{\prime}}$ was assumed, whereby the error associated with the Seltzer correction can be considered to be included in the $F$-factor value [14].

To calculate the $F$ and $M$ factors, a King-plot analysis was used, whereby the isotope shift for a previously measured ionic transition (468.3 nm) in $\mathrm{Ra}^{+}$[8] was plotted against the 714-nm atomic transition, multiplied by the mass-modification factor $\mu^{A, A^{\prime}}=A A^{\prime} /\left(A^{\prime}-A\right)$. From a linear fit of this data, the $F_{714}$

\footnotetext{
${ }^{1} \mathrm{~A}$ rigorous expression for the field-shift component of the isotope shift, $\delta v^{A, A^{\prime}}=\delta v_{\mathrm{FS}}^{A, A^{\prime}}+\delta v_{\mathrm{MS}}^{A, A^{\prime}}$, is given by $\delta v_{\mathrm{FS}}^{A, A^{\prime}}=F \lambda^{A, A^{\prime}}$, whereby the nuclear parameter $\lambda^{A, A^{\prime}}$ contains corrections for higher-order radial moments, $\lambda^{A, A^{\prime}}=\delta\left\langle r^{2}\right\rangle^{A, A^{\prime}}+\frac{C_{2}}{C_{1}} \delta\left\langle r^{4}\right\rangle^{A, A^{\prime}}+\frac{C_{3}}{C_{1}} \delta\left\langle r^{6}\right\rangle^{A, A^{\prime}}+\ldots$ $[24,25]$.
}

and $M_{714}$ factors can be evaluated, using the relation,

$$
\mu^{A, A^{\prime}} \delta v_{714}^{A, A^{\prime}}=\frac{F_{714}}{F_{468}} \mu^{A, A^{\prime}} \delta v_{468}^{A, A^{\prime}}+M_{714}-\frac{F_{714}}{F_{468}} M_{468},
$$

where $M=K_{\mathrm{NMS}}+K_{\mathrm{SMS}}$, the sum of the normal $\left(K_{\mathrm{NMS}}\right)$ and specific $\left(K_{\mathrm{SMS}}\right)$ mass shifts. The normal mass shift was calculated using $K_{\mathrm{NMS}}=v_{0} m_{e}$, where $v_{0}$ is the transition frequency and $m_{e}$ the mass of the electron (in units of $\mathrm{u}$ ). For the 468-nm transition, the specific mass shift $K_{\mathrm{SMS}}=-990(99) \mathrm{GHz}$ and the field-shift factor $F_{468}=-39.813(1990) \mathrm{GHz} \mathrm{fm}^{-2}$ were taken from ab initio many-body calculations performed in Ref. [14].

Figure 4 shows the King plot of $\mu^{A, A^{\prime}} \delta v_{468}^{A, A^{\prime}}$ against $\mu^{A, A^{\prime}} \delta v_{714}^{A, A^{\prime}}$. Figure 4 (insets) presents $\times 100$ magnification to

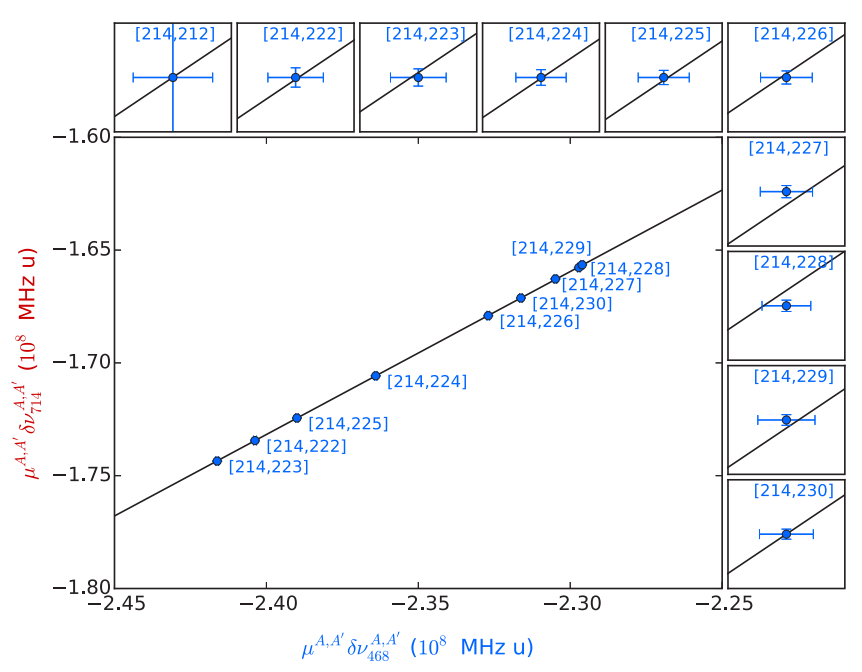

FIG. 4. King-plot analysis for the extraction of the $F$ and $M$ atomic factors for the measured 714-nm transition. Literature values for the 468.3-nm atomic transition in $\mathrm{Ra}^{+}$are taken from Ref. [8]. Insets are $\times 100$ magnification to show the uncertainties on the data values. 
illustrate the uncertainties on the data values for each isotopeshift pair. From the gradient and intercept of the linear fit, the $F$ and $M$ factors for the 714-nm transition were calculated to be

$$
\begin{aligned}
F_{714} & =-28.75(144) \mathrm{GHz} \mathrm{fm}^{-2} \\
M_{714} & =-305(117) \mathrm{GHz} u .
\end{aligned}
$$

This provides $K_{\mathrm{SMS}}=-540(120) \mathrm{GHz}$ u, in good agreement with the experimentally determined $-570(260) \mathrm{GHz}$ u from literature [14]. The field-shift ratio, given by the gradient of the linear fit, was determined to be $F_{714} / F_{468}=0.7222(4)$, which compares well with $0.7218(12)$ from Ref. [14]. The smaller errors are attributed to the smaller uncertainties on our 714-nm transition isotope shifts, in addition to the extended data set, compared to the previous data [8] used in Ref. [14].

An additional scaling factor $\Delta$ (listed in Table I) was applied to the change in mean-square charge radii to account for the nuclear deformation of the heavy radium isotopes, as described in Ref. [14]. For the isotopes ${ }^{231,233} \mathrm{Ra}$, where no scaling factor is given, a value of $\Delta=2.8(1) \%$ is used, based on extrapolation from neighboring isotopes. The uncertainty on the charge radii (associated with the $0.1 \%$ error on the $\Delta$ value) is negligible in comparison to the uncertainty originating from the $F$ and $M$ factors. It accounts for an absolute uncertainty of the change in mean-square charge radii of only $0.002 \mathrm{fm}^{2}$.

\section{The change in mean-square charge radii}

The change in mean-square charge radii for the neutron-rich radium isotopes are presented in Table I. In the same manner as the isotope shifts, the charge-radii values are evaluated relative to ${ }^{214} \mathrm{Ra}$. The statistical uncertainties resulting from the isotope-shift values are shown in parentheses. The systematic uncertainty resulting from the error on the $F$ and $M$ factors are given in brackets. This systematic uncertainty also includes the error resulting from the linear fit in the King-plot analysis. Literature values for the relative charge radii are taken from Ref. [14], using isotope shifts measured from Ref. [8], with the $F$ and $M$ factors for the 468-nm ionic transition as quoted previously.

The change in mean-square charge radii of the francium $(Z=87)$ (red circles) [26-28] and radium $(Z=88)$ (blue markers) $[8,14]$ isotopes are shown in Fig. 5. New values from this work are illustrated by the blue stars. The blue shaded area represents the systematic uncertainty on the radium isotopes, that is propagated from the $5 \%$ uncertainty on $F_{468}$ (and to a much lesser degree, the $10 \%$ uncertainty on $K_{\mathrm{SMS}}$ ) [14]. The statistical uncertainties resulting from the isotope shifts are smaller than the data markers. For the high-resolution measurements of ${ }^{222-232} \mathrm{Ra}$, this represents up to $0.006 \%$ of the relative charge-radii values. Only for the low-resolution measurement of ${ }^{233} \mathrm{Ra}$ does the uncertainty increase to $0.5 \%$.

As previously highlighted [28,29], the radon $(Z=86)$, francium $(Z=87)$, and radium $(Z=88)$ isotopes show a remarkable agreement in their charge radii on both the neutronrich and-deficient edges of the nuclear chart. The odd proton in the $1 h_{9 / 2}$ orbital in the neutron-deficient francium isotopes was suggested to act as a spectator particle in comparison to radon [29], and the behavior of radium on the neutron-rich side is

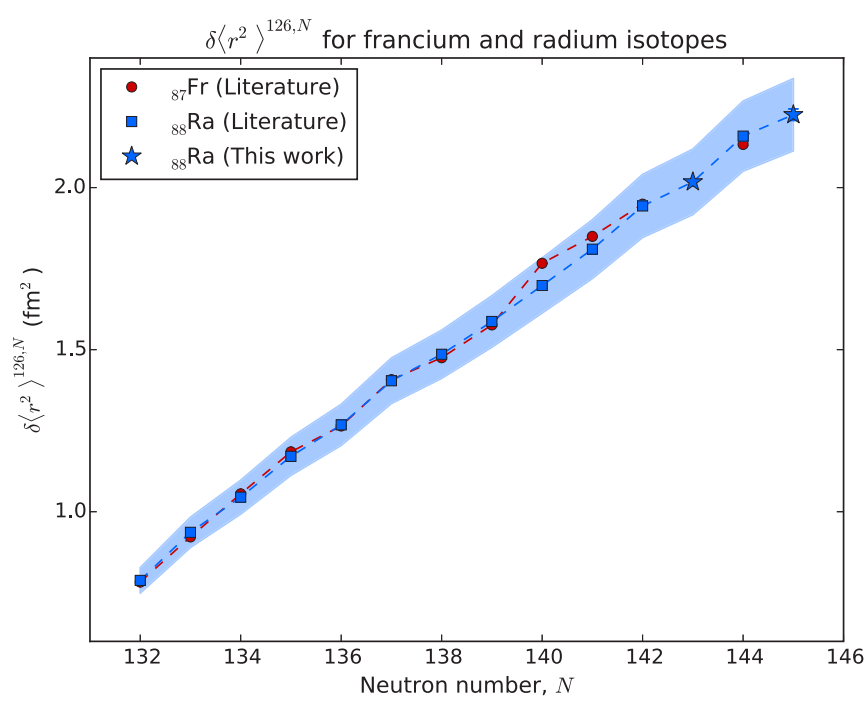

FIG. 5. Change in mean-square charge radii of neutron-rich francium (red circles [26-28]) and radium (blue markers [8,14]) isotopes, relative to $N=126$. The blue shaded area represents the systematic uncertainty on the radium isotopes from the field- and mass-shift factors.

similar. Illustrated in Fig. 5, the extra proton in radium seems to have a negligible effect on the nature of the deformation in these nuclei. The small difference in the charge radii at $N=140,141$ signify the change in the odd-even staggering behavior of the two isotopic chains.

\section{Odd-even staggering}

The odd-even staggering of the change in mean-square charge radii is a well-known effect resulting from the smaller radii of odd- $N$ isotopes in comparison to the mean of their even- $N$ neighbors. This can be quantified by the formalism,

$$
\begin{aligned}
& D\left(N ; \delta\left\langle r^{2}\right\rangle^{126, N}\right) \\
& \quad=(-1)^{N}\left(\delta\left\langle r^{2}\right\rangle^{126, N}-\frac{1}{2}\left(\delta\left\langle r^{2}\right\rangle^{126, N-1}+\delta\left\langle r^{2}\right\rangle^{126, N+1}\right)\right),
\end{aligned}
$$

where $D\left(N ; \delta\left\langle r^{2}\right\rangle\right)<0$ represents an "inversion" of the normal odd-even staggering behavior, i.e., the radius of the odd- $N$ isotope is larger.

Figure 6 presents $D\left(N ; \delta\left\langle r^{2}\right\rangle\right)$ as a function of neutron number for the francium (red circles) [26-28] and radium (blue markers) [8,14] isotopes. Isotopes ${ }^{221-226} \mathrm{Ra}$ display inverted odd-even staggering, with ${ }^{227} \mathrm{Ra}(N=139)$ marking the turning point where normal staggering is resumed. After a small inversion at ${ }^{228} \mathrm{Ra}(N=140)$, the odd-evening staggering returns to normal. New charge-radii measurements of ${ }^{231,233} \mathrm{Ra}$ have allowed $D\left(N ; \delta\left\langle r^{2}\right\rangle\right)$ to be calculated for ${ }^{230-232} \mathrm{Ra}(N=142-$ 144) for the first time. Figure 6 illustrates the continuation of the normal odd-even staggering trend, as shown by the blue stars. The larger uncertainty on $D\left(N ; \delta\left\langle r^{2}\right\rangle^{126,144}\right)$ is a result of the $500 \mathrm{MHz}$ uncertainty on the isotope shift of ${ }^{233} \mathrm{Ra}$.

The inversion of the odd-even staggering behavior has long been associated with octupole deformation and reflectionasymmetric shapes [16]. Figure 6 illustrates this inversion for 


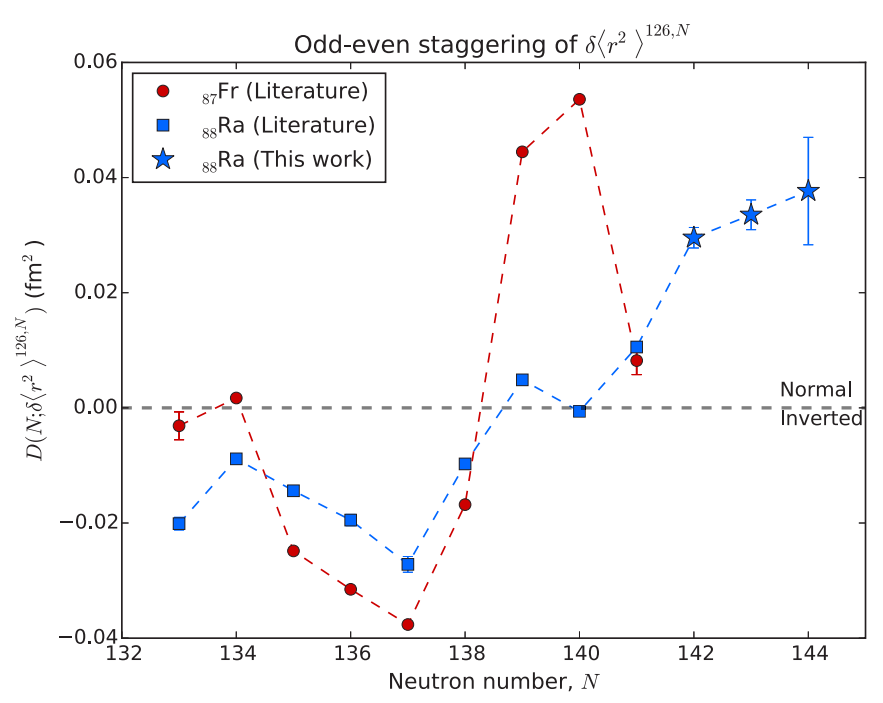

FIG. 6. The odd-even staggering parameter, $D\left(N ; \delta\left\langle r^{2}\right\rangle\right)$ plotted as a function of neutron number for the neutron-rich francium (red circles [26-28]) and radium (blue markers [8,14]) isotopes. $D\left(N ; \delta\left\langle r^{2}\right\rangle\right)$ was calculated from $\delta\left\langle r^{2}\right\rangle$ values using Eq. (3).

isotopes below ${ }^{229} \mathrm{Ra}(N=141)$, consistent with the octupole deformation known to be present in the $220 \leqslant A \leqslant 228$ mass range [30]. Normal odd-even staggering is restored for ${ }^{229} \mathrm{Ra}$ and beyond, where the neutron-rich radium isotopes return to their reflection-symmetric shape. In contrast to the francium odd-even staggering whose $D\left(N ; \delta\left\langle r^{2}\right\rangle\right)$ parameter displays a sudden increase at $N=139,140$, the radium isotopes display no such strong increase, instead continuing a smooth upward trend with increasing neutron number. This increased oddeven staggering agrees with the behavior seen in the binding energies of these isotopes [31]. Here, the enhanced odd-even staggering towards $N=146$ was reproduced by both HartreeFock and Hartree-Fock-Bogoliubov calculations by increasing quadrupole deformation.

\section{E. Electromagnetic moments}

The hyperfine $A$ and $B$ factors of the $7 s 7 p^{3} P_{1}$ atomic state for the odd- $A$ radium isotopes are presented in Table II. For isotopes with multiple scans, the weighted mean and weighted standard deviation were calculated and are presented. For each isotope, the magnetic dipole moment $\mu$ was determined from the hyperfine $A$ factor via the ratio,

$$
\mu=\frac{I A}{I_{\text {ref }} A_{\text {ref }}} \mu_{\text {ref }},
$$

using the reference measurement of ${ }^{225} \mathrm{Ra}$ of $I=1 / 2, \mu=$ $-0.7338(15) \mu_{N}$ [15], and $A\left(7 s 7 p^{3} P_{1}\right)=-9793.9(43) \mathrm{MHz}$ [8]. The magnetic dipole moments for the odd- $A$ radium isotopes are given in Table II. They are presented in comparison to literature [16], showing good consistency and an improved uncertainty for the majority of isotopes. The uncertainty on the magnetic moment is dominated by the systematic error on $\mu_{\text {ref }}$. The statistical uncertainty resulting from our measurement of $A\left({ }^{3} P_{1}\right)$ corresponds to $<0.05 \%$ of the quoted magneticmoment values.

The inclusion of core polarization was shown to be essential to reproduce the magnetic moments of the neutron-rich radium isotopes [8]. In addition, nuclear-theory calculations $[32,33]$ that include static octupole deformation reproduce the magnetic-moment values well. For a detailed review of the magnetic moments of the neutron-rich isotopes, the reader is referred to Refs. $[8,34]$ and references therein.

Similarly, the spectroscopic quadrupole moment $Q_{s}$ was calculated from the hyperfine $B$ factor via the relation,

$$
Q_{s}=Q_{s, \mathrm{ref}} \frac{B}{B_{\mathrm{ref}}},
$$

with reference to the measurements of ${ }^{223} \mathrm{Ra}$ of $Q_{s}=$ $+1.254(66) \mathrm{b}$ [9] and $B\left(7 s 7 p^{3} P_{1}\right)=-470.2(12) \mathrm{MHz}$ [8]. The spectroscopic quadrupole moments are also presented in Table II, in comparison to literature [9]. The statistical and systematic uncertainties are given in parentheses and brackets, respectively. The systematic uncertainty is dominated by the error on $Q_{s}$ of the reference isotope. Agreement with available literature values is very good.

The intrinsic quadrupole moment $Q_{o}$ is related to the spectroscopic quadrupole moment by the equation,

$$
Q_{o}=\frac{(I+1)(2 I+3)}{3\left\langle K^{2}\right\rangle-I(I+1)} Q_{s},
$$

where $K$, the projection of the total spin on the nuclear axis, is set to $K=I$ by assuming a well-deformed state. This assumption is considered valid for the strongly deformed $\left(\beta_{2} \approx\right.$ 0.2 ) heavy Ra nuclei [9], whereby the Coriolis interaction decreases with increasing deformation [35]. Furthermore, the enhanced Coriolis interaction present for $K=1 / 2$ nuclei (i.e.,

TABLE II. Hyperfine $A$ and $B$ factors, magnetic dipole moments, and spectroscopic quadrupole moments for the odd- $A$ radium isotopes

\begin{tabular}{|c|c|c|c|c|c|c|c|}
\hline \multirow[t]{2}{*}{$A$} & \multirow[t]{2}{*}{$I$} & \multirow[t]{2}{*}{$A\left({ }^{3} P_{1}\right)(\mathrm{MHz})$} & \multirow[t]{2}{*}{$B\left({ }^{3} P_{1}\right)(\mathrm{MHz})$} & \multicolumn{2}{|c|}{$\mu\left(\mu_{N}\right)$} & \multicolumn{2}{|c|}{$Q_{s}(\mathrm{~b})$} \\
\hline & & & & This work & Ref. [16] & This work & Ref. [9] \\
\hline 223 & $3 / 2^{+}$ & $+1202.5(3)$ & $-472.1(2)$ & $+0.2703(6)$ & $+0.2705(19)$ & $+1.259(1)[66]$ & $+1.254(3)[66]$ \\
\hline 225 & $1 / 2^{+}$ & $-9795.1(3)$ & - & $-0.7339(15)$ & $-0.7338(15)$ & - & - \\
\hline 227 & $3 / 2^{+}$ & $-1792.8(4)$ & $-593(3)^{\mathrm{a}}$ & $-0.4030(8)$ & $-0.4038(24)$ & $+1.581(9)[80]$ & $+1.58(3)[11]$ \\
\hline 229 & $5 / 2^{+}$ & $+1339.3(7)$ & $-1160(3)$ & $+0.5017(11)$ & $+0.5025(27)$ & $+3.094(8)[160]$ & $+3.09(4)[19]$ \\
\hline 231 & $\left(5 / 2^{+}\right)$ & $-953.5(2)$ & $-1230.7(5)$ & $-0.3572(8)$ & - & $+3.282(1)[170]$ & - \\
\hline
\end{tabular}
${ }^{223-231} \mathrm{Ra}$, compared to literature $[9,16]$. When two errors are presented, the statistical and systematic uncertainties are in parentheses and brackets, respectively.

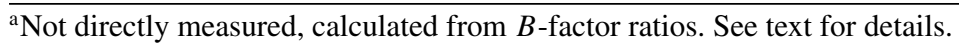




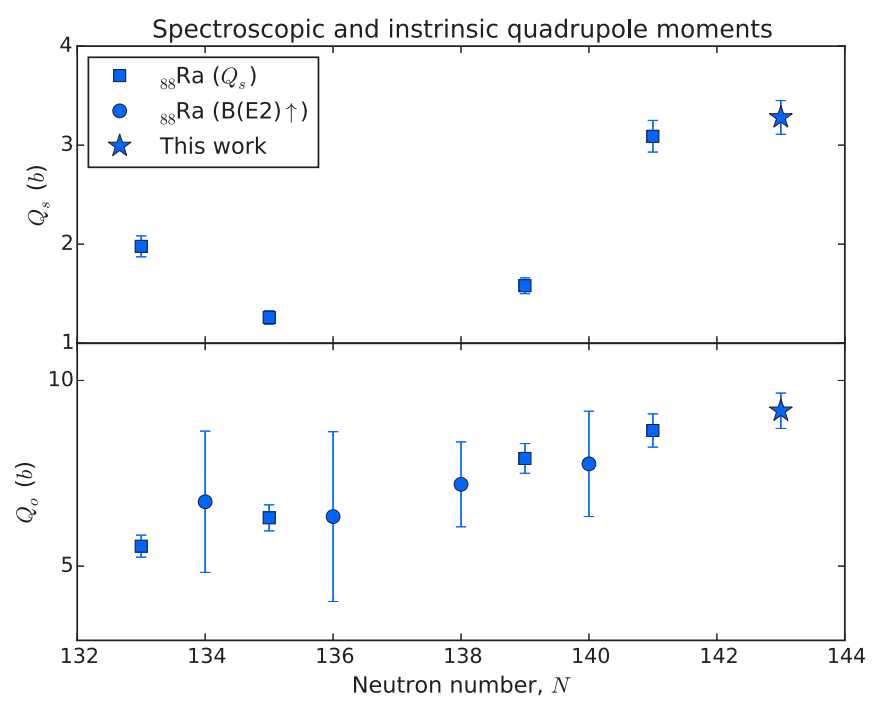

FIG. 7. The (top) spectroscopic and (bottom) intrinsic quadrupole moments of the neutron-rich radium isotopes, from this work (blue star) and literature (blue squares) [9]. $Q_{0}$ moments for the even- $A$ isotopes (blue circles) were calculated from $\mathrm{B}(\mathrm{E} 2) \uparrow$ values [36].

${ }^{225} \mathrm{Ra}$ ) is of no concern, as $Q_{s}$ cannot be measured for this isotope.

Figure 7 shows the spectroscopic quadrupole moments (top) and intrinsic quadrupole moments (bottom). Blue squares present the $Q_{0}$ moments calculated from laser-spectroscopy measurements of the spectroscopic quadrupole moments [9], with the new value for ${ }^{231} \mathrm{Ra}$ illustrated by the blue star. Blue circles present intrinsic quadrupole moments for the even- $A$ isotopes calculated from $\mathrm{B}(\mathrm{E} 2) \uparrow$ values [36] using the formula,

$$
Q_{0}=\sqrt{\frac{16 \pi \mathrm{B}\left(\mathrm{E} 2 ; 0_{1}^{+} \rightarrow 2_{1}^{+}\right)}{5}}
$$

Presented in Fig. 7 (top), the spectroscopic quadrupole moments (ranging from $Q_{s} \approx 1$ to $>3 \mathrm{~b}$ ) illustrate the deformed nature of the neutron-rich radium isotopes, but show no systematic pattern. When viewed in the intrinsic frame in Fig. 7 (bottom), however, they display a surprisingly linear trend of increasing quadrupole deformation with neutron number. This smooth trend is observed irrespective of the filling of the $v\left(2 g_{9 / 2}\right)$ and $v\left(1 i_{11 / 2}\right)$ orbitals and the presence of octupole deformation.

Figure 8 presents the intrinsic quadrupole moments for the even- $Z$ isotopes around the neutron-rich radium isotopes: radon $(Z=86)$ [36,37], thorium $(Z=90)[36,38]$, and uranium $(Z=92)$ [36,39]. The radon data shows no systematic behavior: The small quadrupole moment of ${ }^{221} \mathrm{Rn}$ is attributed to its transitional nature between octupole and quadrupole deformation, where ${ }^{223,225} \mathrm{Rn}$ are known to be reflection symmetric [40]. However, a small offset between the intrinsic quadrupole moments calculated from the $Q_{s}$ and $\mathrm{B}(\mathrm{E} 2) \uparrow$ values is apparent. This could be partly attributed to the electric-field gradient $\left[V_{z z}(0)=-4610 \mathrm{MHz} / \mathrm{b}\right.$, determined from the data [41]] used to calculate $Q_{s \text {,ref for the reference }}$ isotope ${ }^{209} \mathrm{Rn}$. Literature [41-43] does not provide the electricfield gradient used and states that no Sternheimer correction

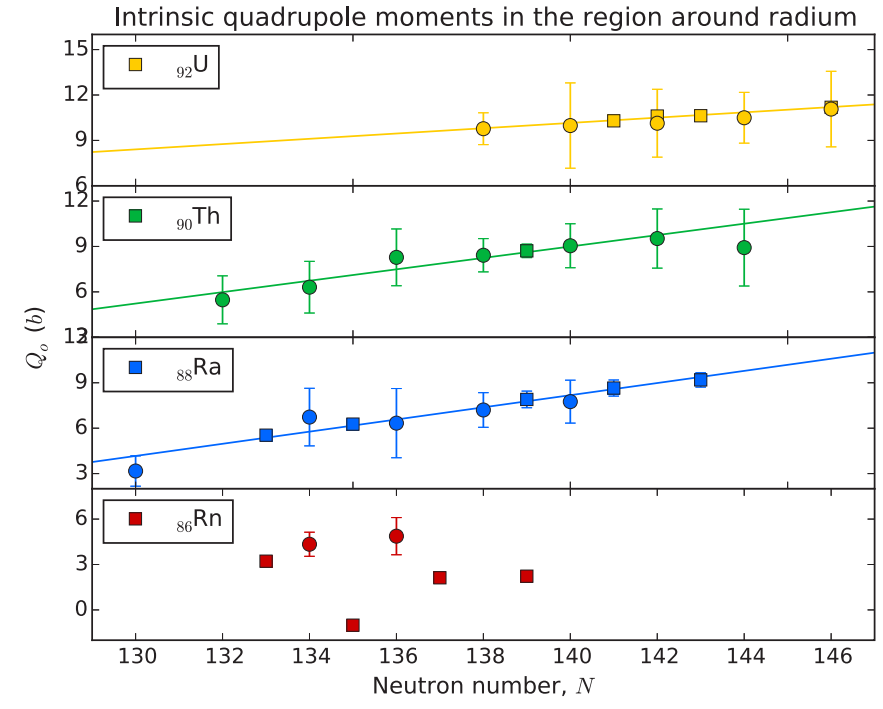

FIG. 8. Intrinsic quadrupole moments for isotopes around radium: radon (red markers) [36,37], radium (blue) [9,36], thorium (green) [36,38], and uranium (yellow) [36,39]. Square markers are calculated from $Q_{s}$ and circular markers are determined from $\mathrm{B}(\mathrm{E} 2) \uparrow$ values.

[44] was applied. Despite this, any reasonable correction (of the order $20 \%$ ) would not be able to account for the smaller moments displayed by ${ }^{219,223,225} \mathrm{Rn}$. New atomic physics calculations are essential to verify $V_{z z}(0)$ and determine if a discrepancy exists.

However, the radium, thorium, and uranium isotopes all display an increasing trend for the intrinsic quadrupole moment in Fig. 8. The smooth trend of the $Q_{0}$ values originating from the $\mathrm{B}(\mathrm{E} 2) \uparrow$ values is expected. Starting at a shell closure, the $\mathrm{B}(\mathrm{E} 2) \uparrow$ values display a characteristic rapid increase with valence neutron number and saturate around the midshell, a result of the enhanced correlations or collectivity (from configuration mixing) as the middle of the neutron shell is approached [35]. However, more surprisingly, the intrinsic quadrupole moments derived from their spectroscopic equivalent fit the isotopic systematics very well. The radium isotopes display a smooth increase in intrinsic quadrupole deformation as the reflection-asymmetric boundary at $N=141$ is passed. There is no sudden decrease in deformation, as observed in the radon isotopes, when reflection symmetry is restored. The increasing magnitude of the intrinsic quadrupole moments adds further experimental support to the calculations presented in Ref. [31].

A linear fit was applied to the data of radium, thorium, and uranium, illustrated by the solid line in Fig. 8. The gradient of each line is $0.40(3), 0.38(6)$, and $0.17(1)$, respectively, which suggests a decreasing gradient with proton number. While the fit follows the radium data well, the quadrupole moment of ${ }^{233} \mathrm{Ra}$ would help determine if the trend continues to increase or saturates at the presently known edge of the nuclear chart. In addition, Coloumb-excitation measurements for more even- $A$ radium isotopes (e.g., ${ }^{220,230,232} \mathrm{Ra}$ ) would provide $\mathrm{B}(\mathrm{E} 2) \uparrow$ values to fully characterize this isotopic chain. The thorium data also agrees with the fit, helped by the large uncertainties on the $\mathrm{B}(\mathrm{E} 2) \uparrow$-derived values. However, with only one intrinsic 
moment derived from the spectroscopic quadrupole moment available $\left({ }^{229} \mathrm{Th}[38]\right)$, more measurements are needed to determine if the odd- $A$ isotopes follow this trend as well as the radium isotopes. The uranium data, with the intrinsic quadrupole moments having been determined from muonic X-ray spectroscopy [39], follow the systematics of the radium and thorium isotopes in a linear fashion.

\section{SUMMARY AND OUTLOOK}

High-resolution collinear resonance ionization spectroscopy was performed on the neutron-rich radium isotopes ${ }^{222-232} \mathrm{Ra}$ for the $7 s^{21} S_{0} \rightarrow 7 s 7 p^{3} P_{1}$ atomic transition. This has allowed the electromagnetic moments of ${ }^{231} \mathrm{Ra}$ to be measured for the first time, yielding $\mu=-0.3572(8) \mu_{N}$ and $Q_{s}=+3.282(170)$ b. Investigation into the intrinsic quadrupole moment displays a surprisingly linear increase with neutron number. Additional low-resolution laser-spectroscopy measurements have enabled determination of the isotope shifts and change in mean-square charge radii of the radium isotopes up to the exotic ${ }^{233} \mathrm{Ra}$. Calculation of the odd-even staggering parameter confirms the continuation of the normal behavior past ${ }^{228} \mathrm{Ra}$ in the reflection-symmetric isotopes, lending further experimental evidence to the increased quadruple deformation in these neutron-rich isotopes.

Future measurement of the quadrupole moment of ${ }^{233} \mathrm{Ra}$ would yield further insight into the nature of the deforma- tion of this isotope, and could determine if the intrinsic deformation reaches saturation for the neutron-rich isotopes. High-resolution measurements of the radon isotopes would also yield interesting information on the $Z=86$ isotopes. Coupled with input from atomic theory on the electric-field gradients and the $F$ and $M$ factors, the quadruple moments and relative charge-radii measurements would allow the transition from reflection-asymmetric to reflection-symmetric shapes to be studied in more detail, thereby allowing the perceived differences between the radon and radium isotopes to be investigated.

\section{ACKNOWLEDGMENTS}

We acknowledge the support of the ISOLDE collaboration and thank the technical teams. This work was supported by ERC Consolidator Grant No. 648381; the Science and Technology Facilities Council Consolidated Grant No. ST/F012071/1, Continuation Grant No. ST/J000159/1, and Ernest Rutherford Grant No. ST/L002868/1; the IUAP-Belgian State Science Policy (BRIX network P7/12), FWO-Vlaanderen (Belgium) and GOA's 10/010 and 10/05 from KU Leuven; and the European Unions Horizon 2020 Framework through ENSAR2 Project No. 654002. K.M.L. acknowledges the support of COFUND, the European Commission Horizon 2020 Marie Skłodowska-Curie Action. We acknowledge the financial aid from the Ed Schneiderman Fund at New York University.
[1] P. A. Butler and W. Nazarewicz, Rev. Mod. Phys. 68, 349 (1996).

[2] P. A. Butler, J. Phys. G: Nucl. Part. Phys. 43, 073002 (2016).

[3] L. P. Gaffney, P. A. Butler, M. Scheck, A. B. Hayes, F. Wenander, M. Albers, B. Bastin, C. Bauer, A. Blazhev, S. Bonig, N. Bree, J. Cederkall, T. Chupp, D. Cline, T. E. Cocolios, T. Davinson, H. De Witte, J. Diriken, T. Grahn, A. Herzan, M. Huyse, D. G. Jenkins, D. T. Joss, N. Kesteloot, J. Konki, M. Kowalczyk, T. Kroll, E. Kwan, R. Lutter, K. Moschner, P. Napiorkowski, J. Pakarinen, M. Pfeiffer, D. Radeck, P. Reiter, K. Reynders, S. V. Rigby, L. M. Robledo, M. Rudigier, S. Sambi, M. Seidlitz, B. Siebeck, T. Stora, P. Thoele, P. Van Duppen, M. J. Vermeulen, M. von Schmid, D. Voulot, N. Warr, K. Wimmer, K. Wrzosek-Lipska, C. Y. Wu, and M. Zielinska, Nature (London) 497, 199 (2013).

[4] P. Möller, A. Sierk, T. Ichikawa, and H. Sagawa, At. Data Nucl. Data Tables 109, 1 (2016).

[5] A. Aas, H. Mach, M. Borge, B. Fogelberg, I. Grant, K. Gulda, E. Hagebo, W. Kurcewicz, J. Kvasil, A. Lindroth, T. Martinez, D. Nosek, B. Rubio, J. Smith, K. Steffensen, J. Tain, O. Tengblad, and T. Thorsteinsen, Nucl. Phys. A 611, 281 (1996).

[6] L. Fraile, A. Aas, M. Borge, B. Fogelberg, L. García-Raffi, I. Grant, K. Gulda, E. Hagebø, W. Kurcewicz, J. Kvasil, G. Løvhøiden, H. Mach, A. Mackova, T. Martínez, B. Rubio, J. Taín, A. Teijeiro, O. Tengblad, and T. Thorsteinsen, Nucl. Phys. A 657, 355 (1999).

[7] L. Fraile, M. Borge, H. Mach, R. Boutami, A. Aas, B. Fogelberg, L. García-Raffi, I. Grant, K. Gulda, E. Hagebø, W. Kurcewicz, J. Kvasil, M. López, G. Løvhøiden, T. Martínez, B. Rubio, J. Taín, and O. Tengblad, Nucl. Phys. A 686, 71 (2001).

[8] K. Wendt, S. A. Ahmad, W. Klempt, R. Neugart, E. W. Otten, and H. H. Stroke, Z. Phys. D 4, 227 (1987).
[9] W. Neu, R. Neugart, E. W. Otten, G. Passler, K. Wendt, B. Fricke, E. Arnold, H. J. Kluge, and G. Ulm, Z. Phys. D 11, 105 (1989).

[10] O. O. Versolato, G. S. Giri, L. W. Wansbeek, J. E. van den Berg, D. J. van der Hoek, K. Jungmann, W. L. Kruithof, C. J. G. Onderwater, B. K. Sahoo, B. Santra, P. D. Shidling, R. G. E. Timmermans, L. Willmann, and H. W. Wilschut, Phys. Rev. A 82, 010501 (2010).

[11] G. S. Giri, O. O. Versolato, J. E. van den Berg, O. Böll, U. Dammalapati, D. J. van der Hoek, K. Jungmann, W. L. Kruithof, S. Müller, M. Nuñez Portela, C. J. G. Onderwater, B. Santra, R. G. E. Timmermans, L. W. Wansbeek, L. Willmann, and H. W. Wilschut, Phys. Rev. A 84, 020503 (2011).

[12] O. Versolato, G. Giri, J. van den Berg, O. Böll, U. Dammalapati, D. van der Hoek, S. Hoekstra, K. Jungmann, W. Kruithof, S. Müller, M. N. Portela, C. Onderwater, B. Santra, R. Timmermans, L. Wansbeek, L. Willmann, and H. Wilschut, Phys. Lett. B 375, 3130 (2011).

[13] O. O. Versolato, L. W. Wansbeek, G. S. Giri, D. J. v. d. H. J. E. van den Berg, K. Jungmann, W. L. Kruithof, C. J. Onderwater, B. K. Sahoo, B. Santra, P. D. Shidling, R. G. Timmermans, L. Willmann, and H. W. Wilschut, Can. J. Phys. 89, 65 (2011).

[14] L. W. Wansbeek, S. Schlesser, B. K. Sahoo, A. E. L. Dieperink, C. J. G. Onderwater, and R. G. E. Timmermans, Phys. Rev. C 86, 015503 (2012).

[15] E. Arnold, W. Borchers, M. Carre, H. T. Duong, P. Juncar, J. Lerme, S. Liberman, W. Neu, R. Neugart, E. W. Otten, M. Pellarin, J. Pinard, G. Ulm, J. L. Vialle, and K. Wendt, Phys. Rev. Lett. 59, 771 (1987).

[16] S. Ahmad, W. Klempt, R. Neugart, E. Otten, P.-G. Reinhard, G. Ulm, and K. Wendt, Nucl. Phys. A 483, 244 (1988). 
[17] U. Dammalapati, K. Jungmann, and L. Willmann, J. Phys. Chem. Ref. Data 45, 013101 (2016).

[18] N. D. Scielzo, J. R. Guest, E. C. Schulte, I. Ahmad, K. Bailey, D. L. Bowers, R. J. Holt, Z.-T. Lu, T. P. O'Connor, and D. H. Potterveld, Phys. Rev. A 73, 010501 (2006).

[19] T. J. Procter, H. Aghaei-Khozani, J. Billowes, M. L. Bissell, F. L. Blanc, B. Cheal, T. E. Cocolios, K. T. Flanagan, H. Hori, T. Kobayashi, D. Lunney, K. M. Lynch, B. A. Marsh, G. Neyens, J. Papuga, M. M. Rajabali, S. Rothe, G. Simpson, A. J. Smith, H. H. Stroke, W. Vanderheijden, and K. Wendt, J. Phys. Conf. Series 381, 012070 (2012).

[20] R. P. de Groote, K. M. Lynch, and S. G. Wilkins, Hyperfine Interact. 238, 5 (2017).

[21] R. P. de Groote, I. Budinčević, J. Billowes, M. L. Bissell, T. E. Cocolios, G. J. Farooq-Smith, V. N. Fedosseev, K. T. Flanagan, S. Franchoo, R. F. Garcia Ruiz, H. Heylen, R. Li, K. M. Lynch, B. A. Marsh, G. Neyens, R. E. Rossel, S. Rothe, H. H. Stroke, K. D. A. Wendt, S. G. Wilkins, and X. Yang, Phys. Rev. Lett. 115, 132501 (2015).

[22] R. P. de Groote, M. Verlinde, V. Sonnenschein, K. T. Flanagan, I. Moore, and G. Neyens, Phys. Rev. A 95, 032502 (2017).

[23] S. Rothe, V. Fedosseev, T. Kron, B. Marsh, R. Rossel, and K. Wendt, Nucl. Instrum. Methods Phys. Res., Sect. B 317, 561 (2013); XVIth International Conference on ElectroMagnetic Isotope Separators and Techniques Related to their Applications, December 2-7, 2012 at Matsue, Japan.

[24] E. C. Seltzer, Phys. Rev. 188, 1916 (1969).

[25] P. Aufmuth, K. Heilig, and A. Steudel, At. Data Nucl. Data Tables 37, 455 (1987).

[26] A. Coc, C. Thibault, F. Touchard, H. Duong, P. Juncar, S. Liberman, J. Pinard, J. Lermé, J. Vialle, S. Büttgenbach, A. Mueller, and A. Pesnelle, Phys. Lett. B 163, 66 (1985).

[27] V. A. Dzuba, W. R. Johnson, and M. S. Safronova, Phys. Rev. A 72, 022503 (2005).

[28] I. Budinčević, J. Billowes, M. L. Bissell, T. E. Cocolios, R. P. de Groote, S. De Schepper, V. N. Fedosseev, K. T. Flanagan, S. Franchoo, R. F. Garcia Ruiz, H. Heylen, K. M. Lynch,
B. A. Marsh, G. Neyens, T. J. Procter, R. E. Rossel, S. Rothe, I. Strashnov, H. H. Stroke, and K. D. A. Wendt, Phys. Rev. C 90, 014317 (2014).

[29] K. M. Lynch, J. Billowes, M. L. Bissell, I. Budinčević, T. E. Cocolios, R. P. De Groote, S. De Schepper, V. N. Fedosseev, K. T. Flanagan, S. Franchoo, R. F. Garcia Ruiz, H. Heylen, B. A. Marsh, G. Neyens, T. J. Procter, R. E. Rossel, S. Rothe, I. Strashnov, H. H. Stroke, and K. D. A. Wendt, Phys. Rev. X 4, 011055 (2014).

[30] R. K. Sheline, Phys. Lett. B 197, 500 (1987).

[31] S. Kreim, D. Beck, K. Blaum, C. Borgmann, M. Breitenfeldt, T. E. Cocolios, A. Gottberg, F. Herfurth, M. Kowalska, Y. A. Litvinov, D. Lunney, V. Manea, T. M. Mendonca, S. Naimi, D. Neidherr, M. Rosenbusch, L. Schweikhard, T. Stora, F. Wienholtz, R. N. Wolf, and K. Zuber, Phys. Rev. C 90, 024301 (2014).

[32] I. Ragnarsson, Phys. Lett. B 130, 353 (1983).

[33] G. Leander and R. Sheline, Nucl. Phys. A 413, 375 (1984).

[34] S. Ahmad, W. Klempt, R. Neugart, E. Otten, K. Wendt, and C. Ekström, Phys. Lett. B 133, 47 (1983).

[35] R. F. Casten, Nuclear Structure from a Simple Perspective (Oxford University Press, Oxford, 2000).

[36] B. Pritychenko, M. Birch, B. Singh, and M. Horoi, At. Data Nucl. Data Tables 107, 1 (2016).

[37] N. Stone, At. Data Nucl. Data Tables 90, 75 (2005).

[38] C. J. Campbell, A. G. Radnaev, and A. Kuzmich, Phys. Rev. Lett. 106, 223001 (2011).

[39] J. D. Zumbro, E. B. Shera, Y. Tanaka, C. E. Bemis, R. A. Naumann, M. V. Hoehn, W. Reuter, and R. M. Steffen, Phys. Rev. Lett. 53, 1888 (1984).

[40] R. Neugart, Hyperfine Interact. 59, 41 (1990).

[41] E. W. Otten (ISOLDE Collaboration), Report No. CERN-EP87-51 (CERN, Geneva, 1987).

[42] W. Borchers, R. Neugart, E. W. Otten, H. T. Duong, G. Ulm, and K. Wendt, Hyperfine Interact. 34, 25 (1987).

[43] R. Neugart, E. Arnold, W. Borchers, W. Neu, G. Ulm, and K. Wendt, AIP Conf. Proc. 164, 126 (1987).

[44] R. Sternheimer, Phys. Rev. 80, 102 (1950). 